\title{
A Case of Drug-induced Linear IgA Bullous Dermatosis
}

\author{
Sofanit A. Dessie ${ }^{1}$, Davinder Singh ${ }^{1}$, Varun Dobariya ${ }^{1}$, Derek Evans ${ }^{2}$, Peimei He ${ }^{3}$ \\ 1. Internal Medicine, Marshall University, Joan C. Edwards School of Medicine, Huntington, USA 2. Medicine, Cabell \\ Huntington Hospital, Huntington, USA 3. Internal Medicine, Marshall University, Huntington, USA
}

Corresponding author: Sofanit A. Dessie, dessie@marshall.edu

\begin{abstract}
Linear immunoglobulin A (IgA) bullous dermatosis (LABD) presents as a rare autoimmune disease that can either occur spontaneously or induced by certain drugs, the most common of which is vancomycin. LABD is a subepidermal blistering disease that is diagnosed by detecting linear IgA bands along the basement membrane. We present a case of a 59-year-old man with worsening blistering skin rash who was treated with vancomycin and piperacillin-tazobactam for pneumonia.
\end{abstract}

Categories: Dermatology, Internal Medicine, Infectious Disease

Keywords: linear iga bullous dermatosis, vancomycin, piperacillin-tazobactam

\section{Introduction}

Intravenous (IV) vancomycin and piperacillin-tazobactam are commonly administered antibiotics to provide a broad spectrum coverage of gram-positive organisms including methicillin-resistant Staphylococcus aureus (MRSA) and gram negatives respectively, frequently given as an empiric therapy for systemic infections including pneumonia [1,2]. Drug-induced linear immunoglobulin A (IgA) bullous dermatosis $(\mathrm{LABD})$ is a rare autoimmune vesiculobullous disorder that affects an older population of adults (mean age of 66.5) [3]. The clinical presentation of LABD may be variable and mimic other blistering disorders such as bullous pemphigoid, cicatricial pemphigoid, dermatitis herpetiformis, erythema multiforme, and toxic epidermal necrolysis (TEN) [4]. In addition to the usual vesiculobullous presentation, vancomycin-induced LABD can also present with erythematous papules, urticarial lesions, eczematous patches, TEN, and erosions [5]. The uncommon nature and variable presentation of LABD makes diagnosis difficult and leads to a delayed recognition of the disease. We present a case where vancomycin and piperacillin-tazobactam were used for presumed pneumonia and likely resulted in drug-induced LABD leading to septic shock and death.

Received 02/25/2020 Review began 02/27/2020 Review ended 03/03/2020 Published 03/04/2020

\section{() Copyright 2020}

Dessie et al. This is an open access article distributed under the terms of the Creative Commons Attribution License CC-BY 4.0., which permits unrestricted use, distribution, and reproduction in any medium, provided the original author and source are credited.

\section{Case Presentation}

A 59-year-old male nursing home resident with a past medical history of quadriplegia secondary to a remote ischemic stroke, chronic obstructive pulmonary disease, coronary artery disease, and chronic kidney disease initially presented to an outside hospital with increased cough and shortness of breath. He was admitted and treated with five days of IV vancomycin and piperacillin-tazobactam for presumed pneumonia. Two weeks after taking these antibiotics, he developed progressive, diffuse, tense rash with blisters that initially started over the scrotum and progressed to involve the oral mucosa, the extremities including palms and soles, chest, torso, and genital regions. He was reported to have persistent symptoms of fever, cough and shortness of breath for which he received another three days of the same antibiotic regimen for persistent pneumonia before he was transferred to our hospital, for management of worsening rash.

He has no known history of drug allergies. Vital signs were significant for blood pressure of $111 / 76 \mathrm{mmHg}$, heart rate of 112 beats per minute, respiratory rate of 22 breaths per minute, temperature of $380 \mathrm{C}$ and saturation of $89 \%$ on room air. Physical examination was significant for bilateral rhonchi on lung examination and approximately fifty 3-4 $\mathrm{mm}$ discrete, denuded vesicles and bullae on skin examination and quadriplegia. Laboratory work was significant for white blood cell (WBC) count of 6.5x109/L, hemoglobin of $8.9 \mathrm{~g} / \mathrm{dL}$, creatinine of 1.26 , sodium of 152 , potassium of 3.8 ; initial blood culture was negative, G-6-PD level was normal, urinalysis was suggestive of urinary traction infection but culture only grew candida species which was likely a contaminant given his chronic indwelling Foley catheter use. Chest X-ray and computed tomography (CT) of the chest were both suggestive of left lower lobe pneumonia. Echocardiogram showed preserved ejection fraction with no wall motion abnormalities. He was initially started on empiric valacyclovir, but skin swab came back negative for herpes and the medication was later discontinued. Vancomycin and piperacillin-tazobactam were empirically started for treatment of presumed health careassociated pneumonia. The last dose of vancomycin was given two days after admission. Five days later, he was found to have six to eight new blisters. A punch skin biopsy showed LABD (Figure 1). He was started on oral prednisone and dapsone. On the eighth day of admission, his rash started to improve with no new blistering. On day 13, he developed fever, tachycardia, and leukocytosis of 17x109/L. Extensively drugresistant Pseudomonas aeruginosa was isolated in blood and urine, which were believed to be from superinfection of his skin lesions, and he was subsequently started on IV meropenem and tobramycin. 


\section{Cureus}

Ultimately, he developed septic shock and the decision was made by his next of kin for hospice and comfort care. He passed away 11 days afterwards.

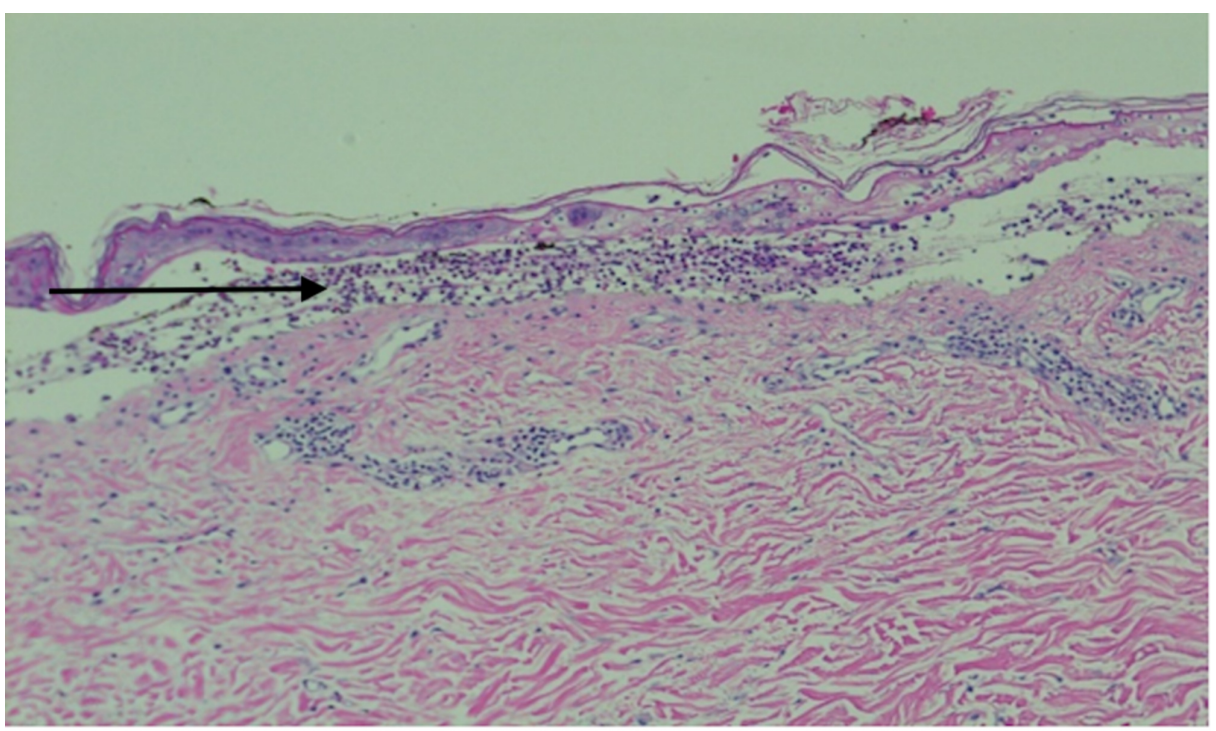

\section{FIGURE 1: Hematoxylin and Eosin stain of the skin biopsy showing inflammatory cells mainly numerous neutrophils within subepidermal separation, as indicated by the arrow}

\section{Discussion}

Although drug-induced LABD is rare, its incidence has been increasing in frequency over the years. Clinicians should have a high index of suspicion for drug-induced LABD at the initial onset of vesicles and bullae after the administration of antibiotics. Multiple drugs have been reported to cause LABD, with vancomycin being the offending agent in $67 \%$ of cases [6]. While multiple studies have determined vancomycin to be the most common drug-induced cause of LABD, we found only one case reporting piperacillin-tazobactam inducing LABD in the absence of vancomycin co-administration. The case report by Adler et al. excluded vancomycin as the culprit drug by only administrating the antibiotic after the onset of the rash and documenting clinical improvement despite ongoing exposure [6]. Numerous reports mention drug-induced LABD but have little research conclusively proving a drug's culpability. For instance, in one case study, 10 patients were on different drug regimens and were examined for LABD. It was concluded that vancomycin was the predominant culprit with lesser offending agents being piperacillin, tazobactam, ciprofloxacin, and trimethoprim-sulfamethoxazole [7]. Hence, we believe our patient's LABD was highly likely to be vancomycin induced than piperacillin-tazobactam, however, the patient's hospital course also included the co-administration of piperacillin-tazobactam making the drug to potentially have a culprit effect. Early diagnosis correlates with decreased mortality and morbidity. Following the initial administration of vancomycin, LABD can occur anytime from one day to one month [8]. In our case, the patient received multiple doses of vancomycin and started to show signs two weeks after antibiotic administration. The diagnosis can be made by direct immunofluorescence searching for linear IgA deposition [9]. In our case report, punch biopsies of the lesions were taken instead confirming the LABD diagnosis (Figure 1).

Our patient represents a likely case of drug-induced LABD given the use of vancomycin and piperacillintazobactam. After strong suspicion of pneumonia, he was prescribed vancomycin and piperacillintazobactam. After 14 days of antibiotic treatment, he developed progressive, diffuse, tense rash with blisters that initially started over the scrotum. After three more days of vancomycin and piperacillin-tazobactam treatment, he was transferred to our hospital and continued to develop new blisters. Eventually, he was started on prednisone and dapsone resulting in short term improvement in his skin rash. Drug-induced LABD is a self-limiting disease and after discontinuing the offending agent, it may resolve spontaneously within a few days [10]. As in our case, the challenge of discontinuing medications is made difficult by patients being on multiple drug regimens. While literature supports vancomycin as the causative agent, we found few reports documenting piperacillin-tazobactam also inducing LABD. Clinicians are tasked with the decision of discontinuing treatment while balancing patient care. In severe cases, prednisone and/or dapsone should be given for 4-6 weeks [10]. In the literature, there are only a few case reports of vancomycin-induced LABD and most cases are resolved within a few days of discontinuing vancomycin along with supportive care. Unfortunately, in our case, the patient continued to receive multiple rounds of vancomycin before it was discontinued, and we believe that impacted his poor outcome. 


\section{Conclusions}

LABD not only mimics various other skin pathologies but also has a common variable presentation. Although drug-induced LABD has a low incidence rate, emerging case reports have shown strong evidence of clinicians overlooking adverse medication reactions. It is important to have a high index of suspicion for potential drug complications and halt poor outcomes by discontinuing the offending agent.

\section{Additional Information \\ Disclosures}

Human subjects: Consent was obtained by all participants in this study. Conflicts of interest: In compliance with the ICMJE uniform disclosure form, all authors declare the following: Payment/services info: All authors have declared that no financial support was received from any organization for the submitted work. Financial relationships: All authors have declared that they have no financial relationships at present or within the previous three years with any organizations that might have an interest in the submitted work. Other relationships: All authors have declared that there are no other relationships or activities that could appear to have influenced the submitted work.

\section{Acknowledgements}

We would like to thank Dr. Jonathan Cuda, Marshall University, Department of Pathology in helping us incorporate the skin punch biopsy images in our case report.

\section{References}

1. Claeys KC, Lagnf AM, Hallesy JA, Compton MT, Gravelin AL, Davis SL, Rybak MJ: Pneumonia caused by methicillin-resistant Staphylococcus aureus: does vancomycin heteroresistance matter?. Antimicrob Agents Chemother. 2016, 60:1708-1716. 10.1128/AAC.02388-15

2. Abdelraouf K, Chavda KD, Satlin MJ, Jenkins SG, Kreiswirth BN, Nicolau DP: Piperacillin-tazobactamresistant/third-generation cephalosporin-susceptible Escherichia coli and Klebsiella pneumoniae isolates: resistance mechanisms and in vitro-in vivo discordance. Int J Antimicrob Agents. 2020, 10.1016/j.ijantimicag.2020.105885

3. Lings K, Bygum A: Linear IgA bullous dermatosis: a retrospective study of 23 patients in Denmark . Acta Derm Venereol. 2015, 95:466-471. 10.2340/00015555-1990

4. Waldman AM, Callen JP: Vancoymycin-induced linear IgA bullous disease presenting as toxic epidermal necrolysis. Clin Exp Dermatol. 2004, 29:633-36. 10.1111/j.1365-2230.2004.01649.x

5. Armstrong AW, Fazeli A, Yeh SW, Mackool BT, Liu V: Vancomycin-induced linear IgA disease manifesting as bullous erythema multiforme. J Cutan Pathol. 2004, 31:393-397. 10.1111/j.0303-6987.2004.00190.x

6. Adler NR, McLean CA, Aung AK, Goh MS: Piperacillin-tazobactam-induced linear IgA bullous dermatosis presenting clinically as Stevens-Johnson syndrome/toxic epidermal necrolysis overlap. Clin Exp Dermatol. 2017, 42:299-302. 10.1111/ced.13030

7. Kakar R, Paugh H, Jaworsky C: Linear Iga bullous disease presenting as toxic epidermal necrolysis: a case report and review of the literature. Dermatology. 2013, 227:209-213. 10.1159/000353584

8. Kang MJ, Kim HO, Park YM: Vanomycin-induced linear IgA bullous dermatosis: a case report and review of the literature. Ann Dermatol. 2008, 20:102. 10.5021/ad.2008.20.2.102

9. Onodera H, Mihm MC Jr, Yoshida A, Akasaka T: Drug-induced linear IgA bullous dermatosis. J Dermatol. 2005, 32:759-764. 10.1111/j.1346-8138.2005.tb00839.x

10. Palmer RA, Ogg G, Allen J, Banerjee A, Ryatt KS, Ratnavel R, Wojnaroska F: Vancomycin-induced linear IgA disease with autoantibodies to BP180 and LAD285. Br J Dermatol. 2001, 145:816-820. 10.1046/j.13652133.2001.04492.x 